\title{
ON HELLY'S PRINCIPLE FOR METRIC SEMIGROUP VALUED BV MAPPINGS OF TWO REAL VARIABLES
}

\author{
M. Balcerzak, S.A. Belov and V.V. Chistyakov
}

\begin{abstract}
We introduce a concept of metric space valued mappings of two variables with finite total variation and define a counterpart of the Hardy space. Then we establish the following Helly type selection principle for mappings of two variables: Let $X$ be $a$ metric space and a commutative additive semigroup whose metric is translation invariant. Then an infinite pointwise precompact family of $X$-valued mappings on the closed rectangle of the plane, which is of uniformly bounded total variation, contains a pointwise convergent sequence whose limit is a mapping with finite total variation.
\end{abstract}

\section{INTRODUCTION}

The interest in the Helly selection principle is natural since it provides an easy and effective way of proving existence theorems in Analysis. The celebrated Helly theorem for monotone functions (Helly [21], see also Natanson [28, Section 8.4]) states that an infinite bounded family of nondecreasing real functions on the closed interval contains a pointwise convergent sequence whose limit is a nondecreasing function. Since a real function on an interval is of bounded (Jordan) variation if and only if it is the difference of two bounded nondecreasing functions (Jordan's decomposition), Helly's theorem implies a selection principle for functions of bounded variation: an infinite bounded family of real functions on the closed interval, which is of uniformly bounded variation, contains a pointwise convergent sequence whose limit is a function of bounded variation.

There are a number of generalisations of the above two selection principles. Let us mention firstly results of Fuchino and Plewik [19] where Helly's theorem for monotone functions is generalised to monotone mappings between linearly ordered sets. Other generalisations are obtained for functions and mappings of one real variable of bounded (generalised) variation. For real valued functions these are due to Musielak and Orlicz [27],

Received 5th February, 2002

The work on the topic of this article has begun when the third author was a visiting scientist at the Institute of Mathematics of the Technical University of Lódź, Poland. The financial support from this institution during the stay of the third author in Lodź, April 2-8, 2000, is gratefully acknowledged. The work of the second and the third authors was also supported by the Ministry of Education of Russian Federation, grant no. E00-1.0-103.

Copyright Clearance Centre, Inc. Serial-fee code: 0004-9727/02 \$A2.00+0.00. 
and for mappings with values in a metric space corresponding results were established by Chistyakov $[9,10,11,12,13,14]$, Chistyakov and Galkin $[16,17]$ and Belov and Chistyakov [7]. One of these generalisations, relevant for our purposes, is presented below as Lemma A. Note that for metric space valued mappings Jordan's decomposition theorem is usually inapplicable, and so, in order to obtain a Helly selection principle, a different technique is used as compared to the real valued case.

There are also counterparts of Helly's selection principle for real functions of two (and more) variables (Adams and Clarkson [1, 2], Hildebrandt [22, III.6.5], Idczak and Walczak [24], Leonov [26]). In Section 2 we recall Helly's theorem for monotone functions of two variables as Lemma B.

Our main goal in this paper is twofold. First, we introduce a concept of metric space valued mappings of two variables with finite total variation (known in the real valued case as mappings from the Hardy space). Second, we establish the following Helly type selection principle for mappings of two variables (Theorem 2): Let $X$ be a metric space and an Abelian additive semigroup whose metric is translation invariant. Then an infinite pointwise precompact family of $X$-valued mappings on the closed rectangle of the plane, which is of uniformly bounded total variation, contains a pointwise convergent sequence whose limit is a mapping with finite total variation. This theorem is based on Helly's theorem for real monotone functions of two variables (Lemma B), Helly's selection principle for metric space valued mappings of one real variable (Lemma A) and a new estimate for mappings of two variables (Theorem 1). Theorem 2 was announced in [14, Theorem 4] and a preliminary version of this paper was published as a preprint in [5].

Our approach to the notion of total variation for metric space valued mappings of several variables is different from the one given by Ambrosio in [3]. The main difference is that we do not assume the target metric space $X$ to be separable and such that bounded closed subsets of $X$ are compact (see [3, Section 1]). Instead, we suppose that an addition operation with natural properties is defined on $X$. Moreover, in the classical case $X=\mathbb{R}$ the Hardy space $B V\left(I_{a}^{b} ; \mathbb{R}\right)$ of functions of two variables with finite total variation, which is a generalisation of the classical space of functions of one variable with finite Jordan variation and of which in turn our space $B V\left(I_{a}^{b} ; X\right)$ is a generalisation, is a Banach algebra as is shown in [15, Theorem 1]; however, we are not aware if this is the case for the space $B V(\Omega ; X)$ with $X=\mathbb{R}$ from [3], since this involves a multiplication of a discontinuous function by a measure, which is a multiplication of distributions. Since the approach in [3, Remark 2.2] gives a generalisation of the notion of essential variation (for definitions see $[6]$ or $[18,4.5 .10])$ for real valued functions of one variable, one cannot expect the compactness criterion from [3, Theorem 2.4] to imply the everywhere convergence of an extracted subsequence (it ensures only the almost everywhere convergence): in fact, we construct a simple example after Theorem 2 in Section 2. 


\section{MAPPINGS WITH FINITE VARIATION: RESULTS}

In this section we review some definitions and known facts and present our main results.

Let $(X, d)$ be a metric space with metric $d$ and $I=[\alpha, \beta] \subset \mathbb{R}$ be a closed interval. The total Jordan variation of a mapping $\varphi: I \rightarrow X$ is the quantity:

$$
V_{\alpha}^{\beta}(\varphi)=V_{1}(\varphi, I)=\sup _{\xi} \sum_{i=1}^{m} d\left(\varphi\left(t_{i}\right), \varphi\left(t_{i-1}\right)\right) \text {, }
$$

where the supremum is taken over all partitions $\xi=\left\{t_{i}\right\}_{i=0}^{m}$ of $I$, that is $m \in \mathbb{N}$ and $\alpha=t_{0}<t_{1}<\cdots<t_{m-1}<t_{m}=\beta$ (for example, [30, Chapter 4, Section 9]). If $V_{\alpha}^{\beta}(\varphi)$ is finite, the mapping $\varphi$ is said to be of finite (Jordan) variation on $[\alpha, \beta]$.

The following Helly type selection principle holds for metric space valued mappings of one real variable:

Lemma A ([9, Theorem 7.1], [10, Theorem 5.1], [7, Theorem 1].) Suppose that $X$ is a metric space and $\Phi$ is an infinite family of mappings from $[\alpha, \beta] \subset \mathbb{R}$ into $X$ such that $\sup _{\varphi \in \Phi} V_{\alpha}^{\beta}(\varphi)$ is finite and the set $\{\varphi(t) \mid \varphi \in \Phi\}$ is precompact in $X$ for each $t \in[\alpha, \beta]$. Then $\Phi$ contains a sequence of mappings which converges in metric $d$ pointwise on $[\alpha, \beta]$ to a mapping $\psi:[\alpha, \beta] \rightarrow X$ such that $V_{\alpha}^{\beta}(\psi)<\infty$.

Now we turn to mappings of two variables.

For points $x, y \in \mathbb{R}^{2}$ we write $x=\left(x_{1}, x_{2}\right), y=\left(y_{1}, y_{2}\right)$ for their respective coordinate representations, and $x \leqslant y$ provided $x_{1} \leqslant y_{1}$ and $x_{2} \leqslant y_{2}$. Given points $a, b \in \mathbb{R}^{2}$ with $a<b$ (that is $a_{1}<b_{1}$ and $a_{2}<b_{2}$ ), we define the basic rectangle (as the domain of most mappings) by $I_{a}^{b}=\left\{x \in \mathbb{R}^{2} \mid a \leqslant x \leqslant b\right\}=\left[a_{1}, b_{1}\right] \times\left[a_{2}, b_{2}\right]$. We note that if $a, b, x$, $y \in \mathbb{R}^{2}, a \leqslant b$ and $x \leqslant y$, then $I_{x}^{y} \subset I_{a}^{b}$ if and only if $a \leqslant x$ and $y \leqslant b$. Such a $I_{x}^{y}$ is called a subrectangle of $I_{a}^{b}$.

To define a notion of finite variation for metric space valued mappings of two variables, we recall the following definition (see [14]):

A triple $(X, d,+)$ is called a metric additive commutative semigroup if $(X, d)$ is a metric space, $(X,+)$ is an additive commutative semigroup and $d$ is translation invariant: $d(u, v)=d(u+w, v+w)$ for all $u, v, w \in X$. Simple examples of metric additive commutative semigroups are: the reals $\mathbb{R}$ with metric $d(u, v)=|u-v|, u, v \in \mathbb{R}$; any normed linear space $(X,\|\cdot\|)$ with metric $d(u, v)=\|u-v\|$ for $u, v \in X$ and a nonempty convex cone $K$ in $X$ (that is, $K \subset X, K+K \subset K$ and $\lambda K \subset K, \lambda \geqslant 0$ ). A more interesting example is the following: If $(X,\|\cdot\|)$ is a real normed linear space, denote by $\mathcal{P}_{\mathrm{cc}}(X)$ the family of all nonempty compact convex subsets of $X$ equipped with the Hausdorff metric $D$ generated by $\|\cdot\|$ (for example, [25]):

$$
D(P, Q)=\max \left\{\sup _{p \in P} \inf _{q \in Q}\|p-q\|, \sup _{q \in Q} \inf _{p \in P}\|p-q\|\right\}, \quad P, Q \in \mathcal{P}_{\mathrm{cc}}(X) .
$$


Then the triple $\left(\mathcal{P}_{\mathrm{cc}}(X), D,+\right)$ is a metric additive commutative semigroup (see [29]) where the addition operation is defined by $P+Q=\{p+q \mid p \in P, q \in Q\}$ for $P$, $Q \in \mathcal{P}_{\mathrm{cc}}(X)$. Recall also that if $X$ is a Banach space, then $\left(\mathcal{P}_{\mathrm{cc}}(X), D,+\right)$ is a complete metric additive commutative semigroup, that is, $\left(\mathcal{P}_{c c}(X), D\right)$ is a complete metric space (see [8, Theorem II-14]).

Let $I_{a}^{b} \subset \mathbb{R}^{2}$ be the basic rectangle, $(X, d,+)$ be a metric additive commutative semigroup and $f: I_{a}^{b} \rightarrow X$ be a given mapping. The mixed difference of the mapping $f$ on a subrectangle $I_{x}^{y}=\left[x_{1}, y_{1}\right] \times\left[x_{2}, y_{2}\right] \subset I_{a}^{b}$ is the value

$$
\operatorname{md}\left(f, I_{x}^{y}\right)=\operatorname{md}\left(f, I_{x_{1}, x_{2}}^{y_{1}, y_{2}}\right)=d\left(f\left(x_{1}, x_{2}\right)+f\left(y_{1}, y_{2}\right), f\left(x_{1}, y_{2}\right)+f\left(y_{1}, x_{2}\right)\right) .
$$

Let $\xi=\left\{t_{i}\right\}_{i=0}^{m}$ be a partition of $\left[a_{1}, b_{1}\right]$ (that is, $m \in \mathbb{N}$ and $a_{1}=t_{0}<t_{1}<\cdots<t_{m-1}$ $<t_{m}=b_{1}$ ) and $\eta=\left\{s_{j}\right\}_{j=0}^{n}$ be a partition of $\left[a_{2}, b_{2}\right]$ (that is, $n \in \mathbb{N}$ and $a_{2}=s_{0}<s_{1}$ $<\cdots<s_{n-1}<s_{n}=b_{2}$ ), so that the rectangles

$$
I_{i, j}=\left[t_{i-1}, t_{i}\right] \times\left[s_{j-1}, s_{j}\right], \quad i=1, \ldots, m, \quad j=1, \ldots, n,
$$

form a partition of $I_{a}^{b}$. The mixed difference of $f$ on $I_{i, j}$ is equal to

$$
\operatorname{md}\left(f, I_{t_{i-1}, s_{j-1}}^{t_{i}, s_{j}}\right)=d\left(f\left(t_{i-1}, s_{j-1}\right)+f\left(t_{i}, s_{j}\right), f\left(t_{i-1}, s_{j}\right)+f\left(t_{i}, s_{j-1}\right)\right) .
$$

The Hardy-Vitali variation $([31,20]$ in the case $X=\mathbb{R})$ of $f: I_{a}^{b} \rightarrow X$ is given by

$$
V_{2}\left(f, I_{a}^{b}\right)=\sup _{\xi, \eta} \sum_{i=1}^{m} \sum_{j=1}^{n} \operatorname{md}\left(f, I_{i, j}\right),
$$

the supremum being taken over all pairs $(\xi, \eta)$ where $\xi=\left\{t_{i}\right\}_{i=0}^{m}$ is a partition of $\left[a_{1}, b_{1}\right]$ and $\eta=\left\{s_{j}\right\}_{j=0}^{n}$ is a partition of $\left[a_{2}, b_{2}\right]$. We define two mappings of one variable as follows: if $x_{2} \in\left[a_{2}, b_{2}\right]$ is fixed, we set $f\left(\cdot, x_{2}\right)(t)=f\left(t, x_{2}\right), t \in\left[a_{1}, b_{1}\right]$, and if $x_{1} \in\left[a_{1}, b_{1}\right]$ is fixed, we set $f\left(x_{1}, \cdot\right)(s)=f\left(x_{1}, s\right), s \in\left[a_{2}, b_{2}\right]$.

We define the total variation of $f: I_{a}^{b} \rightarrow X$ by

$$
T V\left(f, I_{a}^{b}\right)=V_{a_{1}}^{b_{1}}\left(f\left(\cdot, a_{2}\right)\right)+V_{a_{2}}^{b_{2}}\left(f\left(a_{1}, \cdot\right)\right)+V_{2}\left(f, I_{a}^{b}\right)
$$

(for the real-valued case $X=\mathbb{R}$ see [22, III.6.3], [24, Section 2] and [26, Definition 1]), and set $B V\left(I_{a}^{b} ; X\right)=\left\{f: I_{a}^{b} \rightarrow X \mid T V\left(f, I_{a}^{b}\right)<\infty\right\}$. At the end of this section we shall show that the set $B V\left(I_{a}^{b} ; X\right)$ coincides with the Hardy space (Proposition 1 below).

Recall that a real valued function $\nu: I_{a}^{b} \rightarrow \mathbb{R}$ is called totally monotone (in [22, III.4.3] it is called positively monotonely monotone $)$ if $\nu\left(\cdot, a_{2}\right)$ is nondecreasing on $\left[a_{1}, b_{1}\right]$, $\nu\left(a_{1}, \cdot\right)$ is nondecreasing on $\left[a_{2}, b_{2}\right]$ and

$$
\nu\left(x_{1}, x_{2}\right)+\nu\left(y_{1}, y_{2}\right)-\nu\left(x_{1}, y_{2}\right)-\nu\left(y_{1}, x_{2}\right) \geqslant 0
$$

for all $x=\left(x_{1}, x_{2}\right), y=\left(y_{1}, y_{2}\right) \in I_{a}^{b}$ such that $x \leqslant y$. Real totally monotone functions of several variables are thoroughly studied (Antosik [4], Hildebrandt [22], Idczak [23], 
Leonov [26], Young and Young [32]). From this theory we only need a result concerning discontinuity points of these functions (see Lemma $\mathrm{C}$ in Section 3) and the following counterpart of Helly's theorem:

Lemma B ([22, III.6.5], [24, Theorem 3.1], [26, Lemma 3].) An infinite uniformly bounded family of totally monotone functions from $I_{a}^{b}$ into $\mathbb{R}$ contains a sequence which converges pointwise on $I_{a}^{b}$ to a totally monotone function $\nu: I_{a}^{b} \rightarrow \mathbb{R}$.

As in the case of functions of one variable, Jordan's decomposition theorem is valid for real functions of two variables with finite total variation $([\mathbf{2 2}, \mathbf{2 4}, \mathbf{2 6}])$, and so, Lemma $\mathrm{B}$ readily implies a Helly selection principle for these functions.

Our first main theorem will be proved in Section 3:

THEOREM 1. If $(X, d,+)$ is a metric additive commutative semigroup, $a \leqslant x$ $\leqslant y \leqslant b$ in $\mathbb{R}^{2}$ and $f \in B V\left(I_{a}^{b} ; X\right)$, then

(a) $d(f(y), f(x)) \leqslant T V\left(f, I_{x}^{y}\right) \leqslant T V\left(f, I_{a}^{y}\right)-T V\left(f, I_{a}^{x}\right)$;

(b) the function $\nu: I_{a}^{b} \rightarrow \mathbb{R}$ defined by $\nu(x)=T V\left(f, I_{a}^{x}\right), x \in I_{a}^{b}$, is totally monotone.

For real valued functions $f \in B V\left(I_{a}^{b} ; \mathbb{R}\right)$ (of $N$ real variables) the first inequality in Theorem 1(a) was obtained in [26, Corollaries 1 and 5]. The second inequality in Theorem 1(a) is new even for real valued functions, and its counterpart for mappings of one variable is well known. Also, in the real valued case Theorem 1(b) was proved in [22, III.4.3] (for functions of two variables) and [26, Theorem 3] (for functions of $N$ variables).

The second main result is the following Helly type selection principle (its proof is given in Section 3).

THEOREM 2. Let $(X, d,+)$ be a metric additive commutative semigroup and $\mathcal{F}$ be an infinite family of mappings from $I_{a}^{b}$ into $X$ such that $\sup _{f \in \mathcal{F}} T V\left(f, I_{a}^{b}\right)$ is finite and the set $\{f(x) \mid f \in \mathcal{F}\}$ is precompact in $X$ for each $x \in I_{a}^{b}$. Then $\mathcal{F}$ contains a sequence of mappings which converges pointwise on $I_{a}^{b}$ to a mapping $f: I_{a}^{b} \rightarrow X$ such that $T V\left(f, I_{a}^{b}\right)<\infty$.

Now let us show that the Helly selection principle for functions of essentially bounded variation, which gives the almost eveywhere convergence $($ see $[3,6])$, does not in general imply the everywhere convergence of a selected subsequence. Let $I=[0,1], X=\mathbb{R}$, $a_{k}=1 / k$ for $k \in \mathbb{N}$ and $A=\left\{a_{k}\right\}_{k=1}^{\infty}$. Recall that the essential variation $V_{\text {ess }}(\varphi, I)$ of a function $\varphi: I \rightarrow \mathbb{R}$ is given by

$$
V_{\text {ess }}(\varphi, I)=\inf \left\{V_{1}(\psi, I) \mid \psi: I \rightarrow \mathbb{R} \text { and } \psi=\varphi \text { almost everywhere on } I\right\} .
$$

For $n \in \mathbb{N}$ define $\varphi_{2 n-1}(x)=0$ for all $x \in I, \varphi_{2 n}(x)=0$ if $x \neq a_{k}$ for all $k \in \mathbb{N}$, and $\varphi_{2 n}\left(a_{k}\right)=a_{k}$ for $k \in \mathbb{N}$. Clearly, the sequence $\left\{\varphi_{n}\right\}_{n=1}^{\infty}$ is uniformly bounded, 
$V_{1}\left(\varphi_{n}, I\right)=2 a_{k}$ and $V_{\text {ess }}\left(\varphi_{n}, I\right)=0, n \in \mathbb{N}$. If we apply the Helly selection principle for functions $\left\{\varphi_{n}\right\}_{n=1}^{\infty}$, treated as functions of essentially bounded variation, any almost everywhere convergent subsequence of $\left\{\varphi_{n}\right\}_{n=1}^{\infty}$ will do, for example, $\left\{\varphi_{n}\right\}_{n=1}^{\infty}$ itself. But it converges only on $[0,1] \backslash A$ with $A$ having Lebesgue measure zero. The classical Helly selection principle for functions of finite Jordan variation is thus more subtle: it ensures the existence of an everywhere convergent subsequence of $\left\{\varphi_{n}\right\}_{n=1}^{\infty}$, for instance, $\left\{\varphi_{2 n-1}\right\}_{n=1}^{\infty}$. A similar situation holds for functions of two variables: one just has to define $f_{n}: I \times I \rightarrow \mathbb{R}$ by $f_{n}\left(x_{1}, x_{2}\right)=\varphi_{n}\left(x_{1}\right)$ for $x_{1}, x_{2} \in I, n \in \mathbb{N}$.

We shall end this section by showing that the definition of the set $B V\left(I_{a}^{b} ; X\right)$ coincides with the definition of the Hardy space $([\mathbf{1}, \mathbf{2}, \mathbf{2 0}])$ of mappings of two variables with finite total variation (for real valued functions this was proved in [26, Corollary 4]). To do this, we mention some simple properties of metric additive commutative semigroups. If $(X, d,+)$ is a metric additive commutative semigroup, then, due to the translation invariance of $d$ on $X$ and the triangle inequality for $d$, for all $u, v, p, q \in X$ we have:

$$
\begin{aligned}
d(u, v) & \leqslant d(p, q)+d(u+p, v+q), \\
d(u+p, v+q) & \leqslant d(u, v)+d(p, q) .
\end{aligned}
$$

Inequality (5) implies that the addition operation $(u, v) \mapsto u+v$ is a continuous mapping from $X \times X$ to $X$, and more generally, if $u_{n} \rightarrow u, v_{n} \rightarrow v, p_{n} \rightarrow p$ and $q_{n} \rightarrow q$ in $X$ as $n \rightarrow \infty$, then $\lim _{n \rightarrow \infty} d\left(u_{n}+v_{n}, p_{n}+q_{n}\right)=d(u+v, p+q)$.

A partition of $I_{a}^{b}$ is any finite collection $\left\{I_{n}\right\}_{n=1}^{N}$ of subrectangles of $I_{a}^{b}$ (not necessarily of the form (1)) having at most edges or vertices in common such that $I_{a}^{b}=\bigcup_{n=1}^{N} I_{n}$. The Hardy-Vitali variation of $f: I_{a}^{b} \rightarrow X$ (where $X$ is a metric additive commutative semigroup) is defined by the formula: $\widetilde{V}_{2}\left(f, I_{a}^{b}\right)=\sup \sum_{n=1}^{N} \operatorname{md}\left(f, I_{n}\right)$ where the supremum is over all $N \in \mathbb{N}$ and all partitions $\left\{I_{n}\right\}_{n=1}^{N}$ of the rectangle $I_{a}^{b}$. The Hardy space is the set of all mappings $f: I_{a}^{b} \rightarrow X$ for which the variations $V_{a_{1}}^{b_{1}}\left(f\left(\cdot, a_{2}\right)\right), V_{a_{2}}^{b_{2}}\left(f\left(a_{1}, \cdot\right)\right)$ and $\widetilde{V}_{2}\left(f, I_{a}^{b}\right)$ are finite. The coincidence of $B V\left(I_{a}^{b} ; X\right)$ with the Hardy space is established in the following

PROPOSITION 1. For any mapping $f \in B V\left(I_{a}^{b} ; X\right)$ the following equalities hold:

$$
V_{a_{1}}^{b_{1}}\left(f\left(\cdot, a_{2}\right)\right)+V_{a_{2}}^{b_{2}}\left(f\left(a_{1}, \cdot\right)\right)+\widetilde{V}_{2}\left(f, I_{a}^{b}\right)=\sup v(f ; \xi, \eta)=T V\left(f, I_{a}^{b}\right)
$$

where the supremum is taken over all partitions $\xi=\left\{t_{i}\right\}_{i=0}^{m}(m \in \mathbb{N})$ of the interval $\left[a_{1}, b_{1}\right]$ and all partitions $\eta=\left\{s_{j}\right\}_{j=0}^{n}(n \in \mathbb{N})$ of $\left[a_{2}, b_{2}\right]$ and

$$
\begin{aligned}
v(f ; \xi, \eta)=\sum_{i=1}^{m} d( & \left.f\left(t_{i}, a_{2}\right), f\left(t_{i-1}, a_{2}\right)\right)+\sum_{j=1}^{n} d\left(f\left(a_{1}, s_{j}\right), f\left(a_{1}, s_{j-1}\right)\right) \\
& +\sum_{i=1}^{m} \sum_{j=1}^{n} d\left(f\left(t_{i-1}, s_{j-1}\right)+f\left(t_{i}, s_{j}\right), f\left(t_{i-1}, s_{j}\right)+f\left(t_{i}, s_{j-1}\right)\right) .
\end{aligned}
$$


Proof: We shall prove only the first equality since the second one will follow from it. The inequality $\geqslant$ is clear, so let us prove the inequality $\leqslant$. Let us fix $\varepsilon>0$. There exists a partition $\left\{I_{n}^{\varepsilon}\right\}_{n=1}^{N_{\varepsilon}}$ of $I_{a}^{b}$ such that $\tilde{V}_{2}\left(f, I_{a}^{b}\right)-\varepsilon / 3 \leqslant \sum_{n=1}^{N_{\varepsilon}} \operatorname{md}\left(f, I_{n}^{\varepsilon}\right)$. Similarly, there exist a partition $\xi_{\varepsilon}=\left\{t_{i}^{\varepsilon}\right\}_{i=0}^{m_{\varepsilon}}$ of $\left[a_{1}, b_{1}\right]$ and a partition $\eta_{\varepsilon} \stackrel{n=1}{=}\left\{s_{j}^{\varepsilon}\right\}_{j=0}^{n_{\varepsilon}}$ of $\left[a_{2}, b_{2}\right]$ such that the quantities $V_{a_{1}}^{b_{1}}\left(f\left(\cdot, a_{2}\right)\right)-\varepsilon / 3$ and $V_{a_{2}}^{b_{2}}\left(f\left(a_{1}, \cdot\right)\right)-\varepsilon / 3$ can be estimated from above appropriately (see the left hand side inequalities in (6) and (7) below). We extend the edges of all rectangles in the partition $\left\{I_{n}^{\varepsilon}\right\}_{n=1}^{N_{\varepsilon}}$ to the boundary of $I_{a}^{b}$. This gives rise to a partition $\xi_{0}$ of $\left[a_{1}, b_{1}\right]$ and a partition $\eta_{0}$ of $\left[a_{2}, b_{2}\right]$. Set $\xi=\xi_{\varepsilon} \cup \xi_{0}, \eta=\eta_{\varepsilon} \cup \eta_{0}$, suppose that $\xi=\left\{t_{i}\right\}_{i=0}^{m}, \eta=\left\{s_{j}\right\}_{j=0}^{n}$ and let $\left\{I_{i, j} \mid 1 \leqslant i \leqslant m, 1 \leqslant j \leqslant n\right\}$ be a partition of $I_{a}^{b}$ of the form (1) corresponding to $\xi$ and $\eta$. Using the triangle inequality, we find from the above inequalities that

$$
\begin{aligned}
V_{a_{1}}^{b_{1}}\left(f\left(\cdot, a_{2}\right)\right)-\frac{\varepsilon}{3} & \leqslant \sum_{i=1}^{m_{e}} d\left(f\left(t_{i}^{\varepsilon}, a_{2}\right), f\left(t_{i-1}^{\varepsilon}, a_{2}\right)\right) \leqslant \sum_{i=1}^{m} d\left(f\left(t_{i}, a_{2}\right), f\left(t_{i-1}, a_{2}\right)\right) \\
V_{a_{2}}^{b_{2}}\left(f\left(a_{1}, \cdot\right)\right)-\frac{\varepsilon}{3} & \leqslant \sum_{j=1}^{n_{e}} d\left(f\left(a_{1}, s_{j}^{\varepsilon}\right), f\left(a_{1}, s_{j-1}^{\varepsilon}\right)\right) \leqslant \sum_{j=1}^{n} d\left(f\left(a_{1}, s_{j}\right), f\left(a_{1}, s_{j-1}\right)\right) \\
\tilde{V}_{2}\left(f, I_{a}^{b}\right)-\frac{\varepsilon}{3} & \leqslant \sum_{n=1}^{N_{\varepsilon}} \operatorname{md}\left(f, I_{n}^{\varepsilon}\right) \leqslant \sum_{i=1}^{m} \sum_{j=1}^{n} \operatorname{md}\left(f, I_{i, j}\right) .
\end{aligned}
$$

The last inequality in (8) is a consequence of inequalities (4) and (5) and the translation invariance of $d$. To see this, it suffices to consider only two "basic" partitions of $I_{a}^{b}$ of the form:

(i) $I_{a}^{b}=\left(\left[a_{1}, x_{1}\right] \times\left[a_{2}, b_{2}\right]\right) \cup\left(\left[x_{1}, b_{1}\right] \times\left[a_{2}, b_{2}\right]\right)$, and

(ii) $I_{a}^{b}=\left(\left[a_{1}, b_{1}\right] \times\left[a_{2}, y_{2}\right]\right) \cup\left(\left[a_{1}, b_{1}\right] \times\left[y_{2}, b_{2}\right]\right)$.

In case (i) we have:

$$
\begin{aligned}
& d\left(f\left(a_{1}, a_{2}\right)+f\left(b_{1}, b_{2}\right), f\left(a_{1}, b_{2}\right)+f\left(b_{1}, a_{2}\right)\right) \\
& \leqslant d\left(f\left(a_{1}, a_{2}\right)+f\left(b_{1}, b_{2}\right)+f\left(x_{1}, b_{2}\right)+f\left(b_{1}, a_{2}\right), f\left(a_{1}, b_{2}\right)+f\left(b_{1}, a_{2}\right)\right. \\
& \left.\quad+f\left(x_{1}, a_{2}\right)+f\left(b_{1}, b_{2}\right)\right)+d\left(f\left(x_{1}, b_{2}\right)+f\left(b_{1}, a_{2}\right), f\left(x_{1}, a_{2}\right)+f\left(b_{1}, b_{2}\right)\right) \\
& =d\left(f\left(a_{1}, a_{2}\right)+f\left(x_{1}, b_{2}\right), f\left(a_{1}, b_{2}\right)+f\left(x_{1}, a_{2}\right)\right) \\
& \quad+d\left(f\left(x_{1}, a_{2}\right)+f\left(b_{1}, b_{2}\right), f\left(x_{1}, b_{2}\right)+f\left(b_{1}, a_{2}\right)\right)
\end{aligned}
$$

that is, $\operatorname{md}\left(f, I_{a}^{b}\right) \leqslant \operatorname{md}\left(f, I_{a_{1}, a_{2}}^{x_{1}, b_{2}}\right)+\operatorname{md}\left(f, I_{x_{1}, a_{2}}^{b_{1}, b_{2}}\right)$. Case (ii) is similar.

To end the proof, it suffices to add the inequalities (6)-(8). 


\section{PRoOFs of MAIN THEOREMS}

There are two main properties of $V_{2}$; additivity and lower semi-continuity. Additivity means for any partition of $I_{a}^{b}$ of the form (1) we have:

$$
V_{2}\left(f, I_{a}^{b}\right)=\sum_{i=1}^{m} \sum_{j=1}^{n} V_{2}\left(f, I_{i, j}\right)
$$

The (sequential) lower semi-continuity of $V_{2}\left(\cdot, I_{a}^{b}\right)$ means if $f_{n}: I_{a}^{b} \rightarrow X(n \in \mathbb{N})$ is a sequence of mappings which converges pointwise on $I_{a}^{b}$ in metric $d$ to a mapping $f: I_{a}^{b} \rightarrow X$, then

$$
V_{2}\left(f, I_{a}^{b}\right) \leqslant \liminf _{n \rightarrow \infty} V_{2}\left(f_{n}, I_{a}^{b}\right)
$$

The properties of the Jordan variation $V_{1}(\cdot, \cdot)$ (for mappings of one real variable) corresponding to (9) and (10) are very well known (for example, [9] and [10]). It follows from (2) that (9) and (10) also hold for $T V(\cdot, \cdot)$ in place of $V_{2}(\cdot, \cdot)$.

PROOF OF THEOREM 1. (a) To prove the first inequality, we apply inequalities (4), (5), the translation invariance of $d$ and definition (2):

$$
\begin{aligned}
d(f(y), f(x)) \leqslant & d\left(f\left(x_{1}, x_{2}\right)+f\left(x_{1}, x_{2}\right), f\left(x_{1}, y_{2}\right)+f\left(y_{1}, x_{2}\right)\right) \\
& \quad+d\left(f\left(y_{1}, y_{2}\right)+f\left(x_{1}, x_{2}\right)+f\left(x_{1}, x_{2}\right), f\left(x_{1}, x_{2}\right)+f\left(x_{1}, y_{2}\right)+f\left(y_{1}, x_{2}\right)\right) \\
\leqslant & d\left(f\left(x_{1}, x_{2}\right), f\left(y_{1}, x_{2}\right)\right) \\
& \quad+d\left(f\left(x_{1}, x_{2}\right), f\left(x_{1}, y_{2}\right)\right)+d\left(f\left(x_{1}, x_{2}\right)+f\left(y_{1}, y_{2}\right), f\left(x_{1}, y_{2}\right)+f\left(y_{1}, x_{2}\right)\right) \\
\leqslant & V_{x_{1}}^{y_{1}}\left(f\left(\cdot, x_{2}\right)\right)+V_{x_{2}}^{y_{2}}\left(f\left(x_{1}, \cdot\right)\right)+V_{2}\left(f, I_{x}^{y}\right) \\
= & T V\left(f, I_{x}^{y}\right) .
\end{aligned}
$$

To prove the second inequality, we first show that

$$
V_{x_{1}}^{y_{1}}\left(f\left(\cdot, x_{2}\right)\right) \leqslant V_{x_{1}}^{y_{1}}\left(f\left(\cdot, a_{2}\right)\right)+V_{2}\left(f, I_{x_{1}, a_{2}}^{y_{1}, x_{2}}\right)
$$

In fact, applying inequality (4), for any $x_{1} \leqslant s \leqslant t \leqslant y_{1}$ we have:

$$
\begin{aligned}
& d\left(f\left(t, x_{2}\right), f\left(s, x_{2}\right)\right) \leqslant d\left(f\left(t, a_{2}\right), f\left(s, a_{2}\right)\right) \\
& \quad+d\left(f\left(s, a_{2}\right)+f\left(t, x_{2}\right), f\left(s, x_{2}\right)+f\left(t, a_{2}\right)\right) .
\end{aligned}
$$

Now, if $\xi=\left\{t_{i}\right\}_{i=0}^{m}$ is a partition of the interval $\left[x_{1}, y_{1}\right]$, setting $s=t_{i-1}$ and $t=t_{i}$ in (12) and summing over $i=1, \ldots, m$ we find that

$$
\begin{aligned}
\sum_{i=1}^{m} d\left(f\left(t_{i}, x_{2}\right), f\left(t_{i-1}, x_{2}\right)\right) & \leqslant \sum_{i=1}^{m} d\left(f\left(t_{i}, a_{2}\right), f\left(t_{i-1}, a_{2}\right)\right)+\sum_{i=1}^{m} \operatorname{md}\left(f, I_{t_{i-1}, a_{2}}^{t_{i}, x_{2}}\right) \\
& \leqslant V_{x_{1}}^{y_{1}}\left(f\left(\cdot, a_{2}\right)\right)+V_{2}\left(f, I_{x_{1}, a_{2}}^{y_{1}, x_{2}}\right)
\end{aligned}
$$


and the inequality (11) follows. In a similar manner we get the inequality:

$$
V_{x_{2}}^{y_{2}}\left(f\left(x_{1}, \cdot\right)\right) \leqslant V_{x_{2}}^{y_{2}}\left(f\left(a_{1}, \cdot\right)\right)+V_{2}\left(f, I_{a_{1}, x_{2}}^{x_{1}, y_{2}}\right)
$$

Now we use (11), (13), the additivity property $(9)$ of $V_{2}(\cdot, \cdot)$ in the form

$$
V_{2}\left(f, I_{a}^{x}\right)+V_{2}\left(f, I_{x}^{y}\right)+V_{2}\left(f, I_{a_{1}, x_{2}}^{x_{1}, y_{2}}\right)+V_{2}\left(f, I_{x_{1}, a_{2}}^{y_{1}, x_{2}}\right)=V_{2}\left(f, I_{a}^{y}\right)
$$

the additivity property of $V_{1}(\cdot, \cdot)$, and definition (2). These give

$$
\begin{aligned}
T V\left(f, I_{x}^{y}\right)= & V_{x_{1}}^{y_{1}}\left(f\left(\cdot, x_{2}\right)\right)+V_{x_{2}}^{y_{2}}\left(f\left(x_{1}, \cdot\right)\right)+V_{2}\left(f, I_{x}^{y}\right) \\
\leqslant & V_{x_{1}}^{y_{1}}\left(f\left(\cdot, a_{2}\right)\right)+V_{2}\left(f, I_{x_{1}, a_{2}}^{y_{1}, x_{2}}\right)+V_{x_{2}}^{y_{2}}\left(f\left(a_{1}, \cdot\right)\right)+V_{2}\left(f, I_{a_{1}, x_{2}}^{x_{1}, y_{2}}\right) \\
& \quad+\left[V_{2}\left(f, I_{a}^{y}\right)-V_{2}\left(f, I_{a}^{x}\right)-V_{2}\left(f, I_{a_{1}, x_{2}}^{x_{1}, y_{2}}\right)-V_{2}\left(f, I_{x_{1}, a_{2}}^{y_{1}, x_{2}}\right)\right] \\
= & {\left[V_{a_{1}}^{y_{1}}\left(f\left(\cdot, a_{2}\right)\right)+V_{a_{2}}^{y_{2}}\left(f\left(a_{1}, \cdot\right)\right)+V_{2}\left(f, I_{a}^{y}\right)\right] } \\
& \quad-\left[V_{a_{1}}^{x_{1}}\left(f\left(\cdot, a_{2}\right)\right)+V_{a_{2}}^{x_{2}}\left(f\left(a_{1}, \cdot\right)\right)+V_{2}\left(f, I_{a}^{x}\right)\right] \\
= & T V\left(f, I_{a}^{y}\right)-T V\left(f, I_{a}^{x}\right) .
\end{aligned}
$$

(b) Note that the function $\nu\left(\cdot, a_{2}\right)$ is nondecreasing on $\left[a_{1}, b_{1}\right]$, since

$$
\nu\left(t, a_{2}\right)=T V\left(f, I_{a_{1}, a_{2}}^{t, a_{2}}\right)=V_{a_{1}}^{t}\left(f\left(\cdot, a_{2}\right)\right), \quad a_{1} \leqslant t \leqslant b_{1} .
$$

Similarly, $\nu\left(a_{1}, s\right)=V_{a_{2}}^{s}\left(f\left(a_{1}, \cdot\right)\right)$ is nondecreasing with respect to $s \in\left[a_{2}, b_{2}\right]$.

It remains to show that inequality (3) holds. Let $x, y \in I_{a}^{b}$ be such that $x \leqslant y$. Using definition (2) and taking into account (14) and the additivity property (9) of $V_{2}(f, \cdot)$, we have:

$$
\begin{aligned}
& \nu\left(x_{1}, x_{2}\right)+\nu\left(y_{1}, y_{2}\right)-\nu\left(x_{1}, y_{2}\right)-\nu\left(y_{1}, x_{2}\right) \\
&=T V\left(f, I_{a}^{x}\right)+T V\left(f, I_{a}^{y}\right)-T V\left(f, I_{a_{1}, a_{2}}^{x_{1}, y_{2}}\right)-T V\left(f, I_{a_{1}, a_{2}}^{y_{1}, x_{2}}\right) \\
&=\left[V_{a_{1}}^{x_{1}}\left(f\left(\cdot, a_{2}\right)\right)+V_{a_{2}}^{x_{2}}\left(f\left(a_{1}, \cdot\right)\right)+V_{2}\left(f, I_{a}^{x}\right)\right] \\
&+\left[V_{a_{1}}^{y_{1}}\left(f\left(\cdot, a_{2}\right)\right)+V_{a_{2}}^{y_{2}}\left(f\left(a_{1}, \cdot\right)\right)+V_{2}\left(f, I_{a}^{y}\right)\right] \\
&-\left[V_{a_{1}}^{x_{1}}\left(f\left(\cdot, a_{2}\right)\right)+V_{a_{2}}^{y_{2}}\left(f\left(a_{1}, \cdot\right)\right)+V_{2}\left(f, I_{a_{1}, a_{2}}^{x_{1}, y_{2}}\right)\right] \\
&-\left[V_{a_{1}}^{y_{1}}\left(f\left(\cdot, a_{2}\right)\right)+V_{a_{2}}^{x_{2}}\left(f\left(a_{1}, \cdot\right)\right)+V_{2}\left(f, I_{a_{1}, a_{2}}^{y_{1}, x_{2}}\right)\right] \\
&=V_{2}\left(f, I_{a}^{x}\right)+\left[V_{2}\left(f, I_{a}^{x}\right)+V_{2}\left(f, I_{x}^{y}\right)+V_{2}\left(f, I_{a_{1}, x_{2}}^{x_{1}, y_{2}}\right)+V_{2}\left(f, I_{x_{1}, a_{2}}^{y_{1}, x_{2}}\right)\right] \\
&-\left[V_{2}\left(f, I_{a}^{x}\right)+V_{2}\left(f, I_{a_{1}, x_{2}}^{x_{1}, y_{2}}\right)\right]-\left[V_{2}\left(f, I_{a}^{x}\right)+V_{2}\left(f, I_{x_{1}, a_{2}}^{y_{1}, x_{2}}\right)\right] \\
&=V_{2}\left(f, I_{x}^{y}\right) \geqslant 0
\end{aligned}
$$

which completes the proof. 
From the last calculation in the proof of Theorem 1 we see that $V_{2}\left(f, I_{a}^{b}\right)$ is finite if and only if there exists a function $\nu: I_{a}^{b} \rightarrow \mathbb{R}$ satisfying (3) such that $\operatorname{md}\left(f, I_{x}^{y}\right) \leqslant \operatorname{md}\left(\nu, I_{x}^{y}\right)$ for all $x, y \in I_{a}^{b}$ with $x \leqslant y$.

In order to prove Theorem 2, we need one more lemma.

LEMmA C. ([32], [22, Theorem III.5.4], [4].) If a function $\nu: I_{a}^{b} \rightarrow \mathbb{R}$ is totally monotone, then the set of discontinuities of $\nu$ lie on at most a countable set of lines parallel to the coordinate axes.

Proof of Theorem 2. The idea of the proof is taken from [13].

For $f \in \mathcal{F}$ and $x \in I_{a}^{b}$ we set $\nu_{f}(x)=T V\left(f, I_{a}^{x}\right)$. The family of real valued functions $\left\{\nu_{f} \mid f \in \mathcal{F}\right\}$ is infinite, uniformly bounded, and each function $\nu_{f}$ is totally monotone by Theorem 1(b). Applying Lemma B we infer that there exists a sequence $\left\{f_{n}\right\}_{n=1}^{\infty} \subset \mathcal{F}$ and a totally monotone function $\nu: I_{a}^{b} \rightarrow \mathbb{R}$ such that

$$
\lim _{n \rightarrow \infty} \nu_{f_{n}}(x)=\nu(x) \text { for all } x \in I_{a}^{b} .
$$

The function $\nu$, being totally monotone, has, by Lemma $\mathrm{C}$, its discontinuity points on a denumerable number of lines parallel to the coordinate axes.

Denote by $I_{1}^{*}$ the union of the set of all rational points of $\left[a_{1}, b_{1}\right]$, the two-point set $\left\{a_{1}, b_{1}\right\}$ and the set of those points $t \in\left[a_{1}, b_{1}\right]$ for which $\{t\} \times\left[a_{2}, b_{2}\right]$ is a discontinuity line of $\nu$. Similarly, let $I_{2}^{*}$ be the union of the set of all rational points of $\left[a_{2}, b_{2}\right]$, the two-point set $\left\{a_{2}, b_{2}\right\}$ and the set of those points $s \in\left[a_{2}, b_{2}\right]$ for which $\left[a_{1}, b_{1}\right] \times\{s\}$ is a line containing points of discontinuity of $\nu$. Due to the remarks above, the sets $I_{1}^{*}$ and $I_{2}^{*}$ are at most countable, say, $I_{1}^{*}=\left\{t_{i}\right\}_{i=1}^{\infty}$ and $I_{2}^{*}=\left\{s_{j}\right\}_{j=1}^{\infty}$. By virtue of definition (2) and the hypotheses of Theorem 2, the family $\left\{f_{n}\left(t_{1}, \cdot\right)\right\}_{n=1}^{\infty}$ of mappings from $\left[a_{2}, b_{2}\right]$ into $X$ satisfies the conditions of Lemma $A$, since, taking into account inequality (13), we have:

$$
V_{a_{2}}^{b_{2}}\left(f_{n}\left(t_{1}, \cdot\right)\right) \leqslant V_{a_{2}}^{b_{2}}\left(f_{n}\left(a_{1}, \cdot\right)\right)+V_{2}\left(f_{n}, I_{a_{1}, a_{2}}^{t_{1}, b_{2}}\right) \leqslant \sup _{g \in \mathcal{F}} T V\left(g, I_{a}^{b}\right)<\infty
$$

and so, it contains a subsequence denoted by $\left\{f_{n}^{1}\left(t_{1}, \cdot\right)\right\}_{n=1}^{\infty}$ which converges in $X$ pointwise on $\left[a_{2}, b_{2}\right]$. In a similar way, let $\left\{f_{n}^{2}\left(t_{2}, \cdot\right)\right\}_{n=1}^{\infty}$ be a pointwise convergent subsequence of $\left\{f_{n}^{1}\left(t_{2}, \cdot\right)\right\}_{n=1}^{\infty}$, and inductively, given $k \in \mathbb{N}, k \geqslant 2$, denote by $\left\{f_{n}^{k}\left(t_{k}, \cdot\right)\right\}_{n=1}^{\infty}$ a pointwise convergent subsequence of $\left\{f_{n}^{k-1}\left(t_{k}, \cdot\right)\right\}_{n=1}^{\infty}$. Then the diagonal sequence $\left\{f_{n}^{n}\right\}_{n=1}^{\infty} \subset \mathcal{F}$ converges in $X$ pointwise on the set $I_{1}^{*} \times\left[a_{2}, b_{2}\right]$. Similarly (starting from the sequence $\left\{f_{n}^{n}\right\}_{n=1}^{\infty}$ and applying inequality (11)) the above "diagonal arguments" apply to extract a subsequence of $\left\{f_{n}^{n}\right\}_{n=1}^{\infty}$ which converges in $X$ pointwise on the set $\left[a_{1}, b_{1}\right] \times I_{2}^{*}$.

Thus, without loss of generality we may assume that the initial sequence $\left\{f_{n}\right\}_{n=1}^{\infty}$ converges in $X$ pointwise on the set $J=\left(I_{1}^{*} \times\left[a_{2}, b_{2}\right]\right) \cup\left(\left[a_{1}, b_{1}\right] \times I_{2}^{*}\right)$.

It remains to show that the sequence $\left\{f_{n}(x)\right\}_{n=1}^{\infty}$ converges in $X$ at each point $x=\left(x_{1}, x_{2}\right) \in I_{a}^{b} \backslash J$, that is, $x$ is a point of continuity of the function $\nu$ such that its 
coordinates $a_{1}<x_{1}<b_{1}$ and $a_{2}<x_{2}<b_{2}$ are irrational. Let $x \in I_{a}^{b} \backslash J$ be such a point. Given $\varepsilon>0$, there exists a point with rational coordinates $y=\left(y_{1}, y_{2}\right) \in I_{a}^{b}$ such that $y_{1}<x_{1}, y_{2}<x_{2}$ and

$$
|\nu(x)-\nu(y)| \leqslant \varepsilon / 9 .
$$

Choose a number $N_{0}(\varepsilon) \in \mathbb{N}$ with the property:

$$
\left|\nu_{f_{n}}(x)-\nu(x)\right| \leqslant \varepsilon / 9, \quad\left|\nu_{f_{n}}(y)-\nu(y)\right| \leqslant \varepsilon / 9, \quad n \geqslant N_{0}(\varepsilon) .
$$

Applying Theorem 1(a), for all $n \geqslant N_{0}(\varepsilon)$ we have:

$$
\begin{aligned}
d\left(f_{n}(x), f_{n}(y)\right) & \leqslant T V\left(f_{n}, I_{y}^{x}\right) \leqslant \nu_{f_{n}}(x)-\nu_{f_{n}}(y) \\
& \leqslant\left|\nu_{f_{n}}(x)-\nu(x)\right|+|\nu(x)-\nu(y)|+\left|\nu(y)-\nu_{f_{n}}(y)\right| \\
& \leqslant \varepsilon / 3 .
\end{aligned}
$$

Since the sequence $\left\{f_{n}(y)\right\}_{n=1}^{\infty}$ is convergent, there exists a number $N_{1}(\varepsilon) \in \mathbb{N}$ such that $d\left(f_{n}(y), f_{m}(y)\right) \leqslant \varepsilon / 3$ for all $n \geqslant N_{1}(\varepsilon)$ and $m \geqslant N_{1}(\varepsilon)$. It follows that if $n$, $m \geqslant \max \left\{N_{0}(\varepsilon), N_{1}(\varepsilon)\right\}$, then

$$
d\left(f_{n}(x), f_{m}(x)\right) \leqslant d\left(f_{n}(x), f_{n}(y)\right)+d\left(f_{n}(y), f_{m}(y)\right)+d\left(f_{m}(y), f_{m}(x)\right) \leqslant \varepsilon .
$$

Thus, the sequence $\left\{f_{n}(x)\right\}_{n=1}^{\infty}$ is Cauchy in $X$, and since it is also precompact (by the assumption), it is convergent in $X$.

Defining $f(x)=\lim _{n \rightarrow \infty} f_{n}(x)$ in $X$ for all $x \in I_{a}^{b}$ and taking into account the lowersemicontinuity (10) of $T V\left(\cdot, I_{a}^{b}\right)$ we find that

$$
T V\left(f, I_{a}^{b}\right) \leqslant \liminf _{n \rightarrow \infty} T V\left(f_{n}, I_{a}^{b}\right) \leqslant \sup _{g \in \mathcal{F}} T V\left(g, I_{a}^{b}\right)<\infty,
$$

and so, $f \in B V\left(I_{a}^{b} ; X\right)$, which was to be proved.

REMARK. The assumptions in Theorem 2 are exact (see [7, Example 1]).

\section{REFERENCES}

[1] C.R. Adams and J.A. Clarkson, 'On the definitions of bounded variation for functions of two variables', Trans. Amer. Math. Soc. 35 (1933), 824-854.

[2] C.R. Adams and J.A. Clarkson, 'Properties of functions $f(x, y)$ of bounded variation', Trans. Amer. Math. Soc. 36 (1934), 711-730.

[3] L. Ambrosio, 'Metric space valued functions of bounded variation', Ann. Scuola Norm. Sup. Pisa Cl. Sci. 17 (1990), 439-478.

[4] P. Antosik, 'An investigation of the continuity of functions of several variables', (in Russian), Ann. Soc. Math. Polon. Ser. I, Comment. Math. Prace Mat. 10 (1966), 101-104.

[5] M. Balcerzak, S.A. Belov and V.V. Chistyakov, 'On metric space valued mappings of two real variables with finite total variation', (Department of Mathematics, Łódź University, Preprint 2001/01, January 2001, Lódź (Poland), $10 \mathrm{pp}$. (Available on the World Wide Web at the URL: http://imul.uni.lodz.pl/preprints). 
[6] J. Banaś and W.G. El-Sayed, 'Functions of generalized bounded variation', Zeszyty Nauk. Politech. Rzeszowskiej Mat. Fiz. 85 (1991), 91-109.

[7] S.A. Belov and V.V. Chistyakov, 'A selection principle for mappings of bounded variation', J. Math. Anal. Appl. 249 (2000), 351-366.

[8] C. Castaing and M. Valadier, Convex analysis and measurable multifunctions, Lecture Notes in Math. 580 (Springer-Verlag, Berlin, 1977).

[9] V.V. Chistyakov, 'On mappings of bounded variation', J. Dynam. Control Systems 3 (1997), 261-289.

[10] V.V. Chistyakov, 'On the theory of multivalued mappings of bounded variation of one real variable', (in Russian), Mat. Sb. 189 (1998), 153-176; English translation: Sb. Math. 189 (1998), 797-819.

[11] V.V. Chistyakov, 'On mappings of bounded variation with values in a metric space', (in Russian), Uspekhi Mat. Nauk 54 (1999), 189-190; English translation: Russian Math. Surveys 54 (1999), 630-631.

[12] V.V. Chistyakov, 'Mappings of bounded variation with values in a metric space: generalizations', (Pontryagin Conference, 2, Nonsmooth Analysis and Optimization, Moscow, 1998), J. Math. Sci. (New York) 100 (2000), 2700-2715.

[13] V.V. Chistyakov, 'On set-valued mappings of finite generalized variation', (in Russian), Mat. Zametki 71 (2002) (to appear).

[14] V.V. Chistyakov, 'On mappings of finite generalized variation and nonlinear operators', (Real Analysis Exchange 24th Summer Symposium Conference Reports, May 2000, 39-43).

[15] V.V. Chistyakov, 'Superposition operators in the algebra of functions of two variables with finite total variation', Monatsh. Math. (2002) (to appear).

[16] V.V. Chistyakov and O.E. Galkin, 'On maps of bounded $p$-variation with $p>1$ ', Positivity 2 (1998), 19-45.

[17] V.V. Chistyakov and O.E. Galkin, 'Mappings of bounded $\Phi$-variation with arbitrary function $\Phi^{\prime}, J$. Dynam. Control Systems 4 (1998), 217-247.

[18] H. Federer, Geometric measure theory (Springer-Verlag, Berlin, 1969).

[19] S. Fuchino and Sz. Plewik, 'On a theorem of E. Helly', Proc. Amer. Math. Soc. 127 (1999), 491-497.

[20] G.H. Hardy, 'On double Fourier series, and especially those which represent the double zeta-function with real and incommensurable parameters', Quart. J. Math. Oxford 37 (1905/1906), 53-79.

[21] E. Helly, 'Über lineare Funktionaloperationen', Sitzungsber. Naturwiss. Kl. Kaiserlichen Akad. Wiss. Wien 121 (1912), 265-297.

[22] T.H. Hildebrandt, Introduction to the theory of integration (Academic Press, New York and London, 1963).

[23] D. Idczak, 'Functions of several variables of finite variation and their differentiability', Ann. Polon. Math. 60 (1994), 47-56.

[24] D. Idczak and S. Walczak, 'On Helly's theorem for functions of several variables and its applications to variational problems', Optimization 30 (1994), 331-343.

[25] K. Kuratowski, Topology, Vol 1 (Academic Press, 1966). 
[26] A.S. Leonov, 'Remarks on the total variation of functions of several variables and on a multidimensional analogue of Helly's choice principle', (in Russian), Mat. Zametki 63 (1998), 69-80; English translation: Math. Notes 63, (1998), 61-71.

[27] J. Musielak and W. Orlicz, 'On generalized variations I', Studia Math. 18 (1959), 11-41.

[28] I.P. Natanson, Theory of functions of a real variable (Frederick Ungar, New York, 1965).

[29] H. Rådström, 'An embedding theorem for spaces of convex sets', Proc. Amer. Math. Soc. 3 (1952), 165-169.

[30] L. Schwartz, Analyse Mathématique, Vol. 1 (Hermann, Paris, 1967).

[31] G. Vitali, 'Sui gruppi di punti e sulle funzioni di variabili reali', Atti Accad. Sci. Torino 43 (1908), 75-92.

[32] W.H. Young and G.C. Young, 'On the discontinuities of monotone functions of several variables', Proc. London Math. Soc. 22 (1924), 124-142.

Institute of Mathematics

Lódź Technical University

al. Politechniki 11

I-2, 90-924 Lódź

and

Faculty of Mathematics

Lódź University

ul. Banacha 22

90-238 Lódź

Poland

e-mail: mbalce@krysia.uni.lodz.pl

Department of Mathematics

University of Nizhny Novgorod

Gagarin Avenue 23

Nizhny Novgorod 603950

Russia

e-mail: chistya@mm.unn.ac.ru

chistya@mm.unn.ru
Department of Mathematics

University of Nizhny Novgorod

Gagarin Avenue 23

Nizhny Novgorod 603950

Russia

e-mail: belov@vmk.unn.ru 